\title{
Eficacia y seguridad de liraglutida para la disminución de peso en adultos: revisión sistemática
}

\author{
Efficacy and safety of liraglutide for weight loss in adults: \\ systematic review
}

\author{
Hernández Rodríguez DC, ${ }_{1,2}$ Monroy Sánchez DC, , ${ }^{2}$ Porras Ramírez A., 4
}

\author{
${ }^{1}$ Médico y cirujano, Universidad El Bosque. Candidata a magíster \\ en epidemiología. Bogotá, Colombia. \\ ${ }^{2}$ Grupo de medicina comunitaria y salud colectiva, Universidad El \\ Bosque. Maestría en Epidemiología. Bogotá, Colombia. \\ ${ }^{3}$ Psicóloga especialista en psicología educativa, candidata a magíster \\ en epidemiología. Bogotá, Colombia. \\ ${ }^{4}$ Coordinación de epidemiología e investigaciones, Los Cobos \\ Medical Center. Bogotá, Colombia. \\ Autor de correspondencia: \\ Diana Carolina Hernández Rodríguez \\ Correo electrónico: dianahernandez12@hotmail.com \\ Fecha de recepción: 21/08/2020 \\ Fecha de aceptación: 16/02/2021
}

\section{Resumen}

Objetivo: evaluar la eficacia y seguridad de liraglutida como medicamento coadyuvante para disminuir el índice de masa corporal (IMC) en personas con sobrepeso y obesidad a nivel mundial.

Materiales y métodos: se realizó la búsqueda de estudios en las bases de datos (TRIP, Embase, Pubmed, Scopus, Epistemonikos, Cochrane y ClinicalKey), cuyo desenlace por evaluar sea la disminución de peso con el uso de liraglutida. Lo anterior se realizó mediante la herramienta PRISMA 2009 con el fin de obtener una revisión sistemática.

Resultados: de un total de 74 estudios, se incluyeron 3 ensayos clínicos aleatorizados que evaluó un total de 4281 participantes. Se evaluó como desenlace principal la disminución de peso y los eventos adversos presentados fueron los desenlaces secundarios. Se realizó un metaanálisis cuyo reporte de pérdida de peso de -5,22 (intervalo de confianza [IC] del $95 \%$ : $-5,95,-4,50)$ y el índice de inconsistencia $\left(\mathrm{I}^{2}\right)$ mostró alta heterogeneidad, al igual que la metarregresión realizada. Los eventos adversos se evidenciaron con mayor frecuencia en el grupo de liraglutida, y los eventos gastrointestinales fueron los más reportados.

Conclusión: los resultados individuales en los estudios demuestran que el uso de liraglutida disminuye el peso. Sin embargo, en esta revisión sistemática se evidencia que no hay una significancia estadística de la evidencia por heterogeneidad de los estudios evaluados.

Palabras clave: liraglutida, sobrepeso, obesidad, eficacia, seguridad.

\section{Abstract}

Objective: To evaluate the efficacy and safety of liraglutide as a coadjuvant medication to reduce the body mass index (BMI) in overweight (BMI: $25-30 \mathrm{~kg} / \mathrm{m}^{2}$ ) and obesity (BMI: > $30 \mathrm{~kg} / \mathrm{m}^{2}$ ) worldwide.

Materials and methods: A search of studies was carried out in several databases (TRIP, Embase, Pubmed, Scopus, Epistemonikos, Cochrane and ClinicalKey), the outcome evaluated was the decrease in weight with the use of liraglutide. We use the PRISMA 2009 tool in order to obtain a systematic review.

Results: A total of 74 studies, 3 randomized clinical trials were included, evaluating 4281 participants. Weight loss was the main outcome, and adverse events were secondary outcomes. Meta-analysis was carried out with a weight loss report of -5.22 (95\% confidence interval [CI]: - 5.95, -4.50), inconsistency index $\left(\mathrm{I}^{2}\right)$ with high heterogeneity, as well as the metaregression performed. Adverse events are evidenced more frequently in the liraglutide group, Gastrointestinal events were the most reported.

Conclusion: The individual results in the studies demonstrate that the use of liraglutide reduces weight. However, this systematic review shows that there is no statistical significance of the evidence for heterogeneity of the studies evaluated.

Keywords: Liraglutide; Overweight; Obesity; Efficacy, Safety. 


\section{Introducción}

La prevalencia de sobrepeso y obesidad a nivel mundial viene en aumento. La Organización Mundial de la Salud (OMS) reportó que: "en 2016 el 39 \% de las personas adultas de 18 o más años tenía sobrepeso, y el $13 \%$ era obesa” (1). En América Latina y el Caribe, por ejemplo, por cada persona que sufre de hambre, 6 sufren de sobrepeso u obesidad (2). Adicional a esto, se observa cómo el sobrepeso y la obesidad afectan a cualquier grupo etario, aunque presenta una diferencia entre resultados por sexo: las mujeres son las más afectadas, en un 27,9\%; mientras que se presentan en el 20,2\% de los hombres (2).

El principal factor que predomina para el inicio del sobrepeso u obesidad en América Latina y el Caribe es el consumo de productos procesados que no son acompañados con una actividad física que ayude con el metabolismo del individuo. Los problemas económicos, sociales, demográficos y ambientales también son factores de riesgo que influyen en la salud y los estilos de vida sana (3).

A nivel mundial y en la región de las Américas, durante las últimas décadas se ha reportado un incremento notorio de las enfermedades crónicas no transmisibles (ECNT) que se ven directamente relacionadas con el incremento de peso, lo que aumenta la morbimortalidad en las personas. Así mismo, la mortalidad está relacionada con patologías como la cardiopatía isquémica, la enfermedad cerebrovascular, la diabetes mellitus tipo 2 (DM2) y la hipertensión arterial (4-6). No obstante, en cuanto a la morbilidad, las patologías más relevantes son la apnea del sueño, la osteoartritis y la posibilidad de cáncer de esófago, colon, recto, hígado, vesícula biliar, páncreas, riñón, linfoma no Hodgkin o mieloma múltiple (7).

Ahora bien, esta condición de aumento de peso en general trae consecuencias sociales y mentales que perjudican la vida del paciente luego de sufrir baja autoestima, depresión y ansiedad, que también dificultan la interactividad y participación con el mundo exterior debido a los estándares físicos que se plantean en la sociedad y generan, por ende, una discriminación social $(8,9)$. Sin embargo, el paciente no es el único afectado en su entorno, sino también las entidades de salud pública, debido al tratamiento y la rehabilitación que se debe ejercer con las patologías secundarias al aumento de peso $(7,10)$.

Según estudios, se ha demostrado que al disminuir entre el $5 \%$ y el $10 \%$ del peso corporal, el paciente reduce patologías como las mencionadas anteriormente, lo que mejora su calidad de vida $(11,12)$; por esta razón, es importante realizar una adecuada prevención primaria. Para ello, se debe tener en cuenta que el tratamiento para el sobrepeso y la obesidad requiere de un enfoque multidisciplinario con hábitos saludables que incluyan cambios en la dieta y el aumento de la actividad física (13, 14). Según la OMS, se debe realizar hasta 150 minutos semanales de actividad física intensa aeróbica y 300 minutos de ejercicio a la semana, realizando actividad física moderada aeróbica (15).
No obstante, el paciente puede recibir como tratamiento coadyuvante el uso de fármacos y procedimientos quirúrgicos como la cirugía bariátrica (3). Estos tratamientos generan cambios en los mecanismos fisiopatológicos de la obesidad, el volumen gástrico, la motilidad gástrica y la saciedad $(16,17)$.

Dentro de los medicamentos que pueden generar dichos cambios se encuentra la liraglutida, agonista del receptor del polipéptido 1 similar a glucagón (GLP-1). Se administra por vía subcutánea en el abdomen, el muslo o la parte superior del brazo (18). Se usa como complemento para lograr el control glucémico en adultos con antecedente de DM2, para disminuir el peso como terapia coadyuvante con dieta y ejercicio en personas con IMC $\geq 30 \mathrm{~kg} / \mathrm{m}^{2}$ o IMC $\geq 27$ con comorbilidades, y para reducir la mortalidad cardiovascular en pacientes con DM2 que también tienen enfermedad cardiovascular (19), lo anterior es aprobado por la Food and Drug Administration (FDA) y en Colombia por Instituto Nacional de Vigilancia de Medicamentos y Alimentos (Invima) $(20,21)$.

El objetivo de este estudio es evaluar la eficacia y seguridad de la liraglutida como medicamento coadyuvante para disminuir el índice de masa corporal (IMC) en personas mayores de 18 años con sobrepeso (IMC: $25-30 \mathrm{~kg} / \mathrm{m}^{2}$ ) y obesidad (IMC: $>30 \mathrm{~kg} / \mathrm{m}^{2}$ ) mediante una revisión sistemática de la literatura.

\section{Metodología}

Esta revisión sistemática se preparó utilizando las guías de informes de PRISMA para revisiones sistemáticas.

\section{Población}

Pacientes mayores de 18 años con sobrepeso (IMC: 25-30 $\mathrm{kg} / \mathrm{m}^{2}$ ) con antecedentes relacionados como presión arterial alta, hiperlipidemia o apnea del sueño y obesidad (IMC: $>30$ $\mathrm{kg} / \mathrm{m}^{2}$ ) sin uso de otro tipo de medicamentos que ayuden a disminuir de peso.

\section{Intervención y comparador}

Uso de liraglutida en dosis de 3,0 mg comparada con placebo.

\section{Desenlaces}

Se midieron los desenlaces primarios y secundarios con el fin de evaluar la efectividad y la seguridad de liraglutida.

\section{Desenlace primario}

Disminución de peso con el uso de liraglutida como tratamiento coadyuvante, uso mínimo de 20 semanas hasta 52 semanas.

\section{Desenlaces secundarios}

Eventos adversos, leves o graves, secundarios al uso de liraglutida. 


\section{Contexto}

Esta revisión consideró todos los estudios realizados en todo el mundo en relación con la evaluación de liraglutida como tratamiento coadyuvante para disminuir peso en personas con sobrepeso y obesidad.

\section{Tipos de estudio}

Esta revisión consideró todos los estudios con diseños comparativos, como los ensayos controlados aleatorizados (ECA), estudios cuasiexperimentales y revisiones sistemáticas, en los cuales se evalúa como desenlace principal la disminución de peso con uso de liraglutida frente a placebo. Adicionalmente, se utilizó una búsqueda de bola de nieve con los resultados encontrados.

\section{Estrategia de búsqueda}

En la estrategia de búsqueda se evaluaron estudios publicados en las siguientes bases de datos: Pubmed, ClinicalKey, Trip, Scopus, Epistemonikos, Cochrane y Embase. Se utilizó la estrategia PICO (Population, Intervention,Comparison and Outcome) y palabras claves establecidas en términos DeCS y MeSH.

- Criterios de inclusión: estudios publicados en inglés, español o portugués, realizados entre el 2012 y el 2019, con texto completo en las bases de datos, cuyo principal desenlace a evaluar fue la disminución del IMC con el uso de liraglutida.

- Criterios exclusión: estudios en los cuales se evalúa como desenlace la intervención de liraglutida en pacientes con diabetes mellitus (DM).

\section{Búsqueda en revistas especializadas}

Cochrane: 0 Cochrane Reviews matching Efficacy and safety of liraglutide as a coadjuvant treatment to reduce the body mass index in Title Abstract Keyword Did you mean: efficacity inefficacy and Cochrane Database of Systematic Reviews Issue 8 of 23, August 2019.

Prospero: Efficacy and safety of liraglutide as a coadjuvant treatment to reduce the body mass index 0 records found for Efficacy and safety of liraglutide as a coadjuvant treatment to reduce the body mass index .23, August 2019.

La fecha de la última búsqueda fue el 23 de agosto de 2019 (la estrategia de búsqueda completa se encuentra disponible previa solicitud a los autores).

\section{Selección de los estudios}

Siguiendo el procedimiento de búsqueda anterior, todas las citas identificadas se cargaron en una base de datos generada en Excel. Los registros obtenidos en la búsqueda sistemática fueron evaluados en un inicio por títulos y resúmenes, de esta forma se eliminaron duplicados. Para estos artículos se obtuvo el texto completo, posteriormente se realizó la valoración de sesgo de cada estudio mediante la herramienta Revi- sed Cochrane risk-of-bias tool for randomized trials (RoB 2), clasificándolo como: "bajo riesgo", "alto riesgo" o "riesgo poco claro“. La calidad metodológica de esta revisión se valoró utilizando escala GRADE. Cada fase fue realizada por dos revisores ( $\mathrm{CH}$ y DM) de forma independiente y ante cualquier desacuerdo se resolvió con un tercer revisor.

\section{Análisis de datos}

Las estadísticas fueron calculadas en el software Review Manager 5. Se utilizó un modelo de inversa de la varianza con efecto aleatorio para variables continuas. Para las variables dicotómicas se utilizó un modelo de Mantel-Haenszel de efecto aleatorio, para calcular el riesgo relativo (RR) con un intervalo de confianza del $95 \%$ (IC) para cada estudio y esta información se usó para realizar el correspondiente metaanálisis y la meta regresión (22).

En general, se consideraron los valores $I^{2}$ mayores del $40 \%$ con heterogeneidad estadística (22).

\section{Síntesis de datos}

Se usó la síntesis narrativa para describir los resultados de los estudios en los cuales el metaanálisis no era clínicamente significativo. Los resultados de los estudios incluidos en el metaanálisis se presentaron por medio del diagrama de bosque.

\section{Resultados}

Con la búsqueda sistemática se identificaron 3 estudios que cumplían los criterios de elegibilidad.

\section{Resultados de la búsqueda}

Se identificó un total de 74 estudios no duplicados. De dichos estudios encontrados al evaluar a través de los títulos/ resúmenes, se excluyeron 66 estudios porque no evaluaban el desenlace de interés, se usaba la liraglutida como tratamiento de antecedente de DM o no presentaban claridad en la metodología aplicada; por lo cual se analizaron los documentos de 8 artículos considerados como relevantes y potenciales estudios a ingresar en el análisis. Se excluyeron 5 estudios, entre los que se encontraron estudios secundarios y un estudio complementario a un estudio principal (Figura 1).

Se realizó un metaanálisis para el desenlace principal a los estudios de Astrup, 2012 (23), Blackman, 2016 (24) y PiSunyer, 2015 (25); sin embargo, este último estudio dado a la diferencia de tamaño de muestra comparado con los estudios de Astrup y Blackman, se decidió no ingresarlo en el metaanálisis de los desenlaces secundarios por la alta heterogeneidad (Tabla 1).

\section{Exclusión de los estudios}

Las razones para la exclusión de los últimos 5 estudios fueron las siguientes: 3 de estos estudios son secundarios 
Figura 1. Proceso de selección de estudios incluidos en la revisión sistemática.

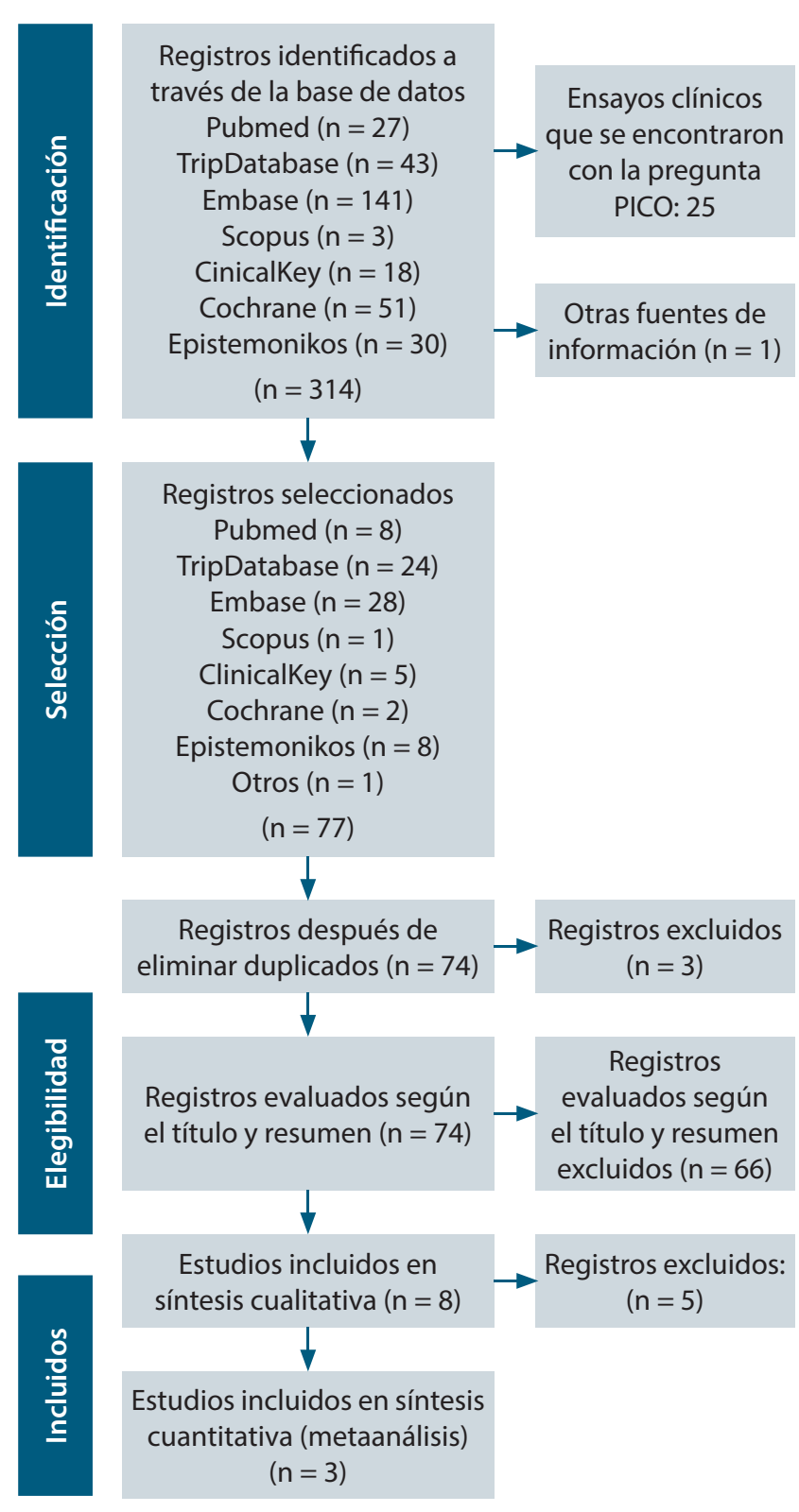

Elaboración propia a partir de datos publicados siguiendo las recomendaciones PRISMA.

basados en los estudios incluidos en esta revisión sin previa revisión de la literatura, sin descartar de este modo el análisis de sesgo y la metodología; y 1 de los estudios fue un estudio piloto con tamaño de muestra pequeño. El último estudio se excluyó por ser parte de la etapa inicial de "Safety, tolerability and sustained weight loss over 2 years with the once-daily human GLP-1 analog, liraglutide", por lo cual generó sesgo de información por una posible duplicación de datos en diferentes valoraciones.

\section{Sesgos incluidos en los estudios}

En los tres ECA incluidos (23-25) se evaluaron los siguientes dominios: sesgo derivado del proceso de aleatorización, sesgo debido a desviaciones de las intervenciones previstas, sesgo debido a datos faltantes de los desenlaces, sesgos en la medición del desenlace y sesgos en la selección de los resultados reportados. En todos los estudios se presentó bajo riesgo de sesgo.

La calidad de la evidencia en general se clasificó como baja, dado por los estándares GRADE; lo anterior se produjo por la imprecisión en algunos enlaces evaluados debido a un amplio IC reflejado, el bajo número de ensayos en los que se basa esta revisión y la alta heterogeneidad en los estudios (26).

\section{Pérdida de peso}

Se realizó un metaanálisis con los 3 ECA incluidos y se reportó una pérdida de peso de $-5,22 \mathrm{~kg}$ IC $95 \%$ : $-5,95,-4,50$ ) con el uso de liraglutida frente a placebo (chi cuadrado $\left[\chi^{2}\right]=$ 11,89; $p=0,003$; índice de inconsistencia $\left[\mathrm{I}^{2}\right]=83 \%$ ); la heterogeneidad posiblemente fue por el tamaño de muestra de cada estudio (Figura 2).

Se realizó una meta regresión. En la Figura 3 cada círculo representa uno de los estudios y el tamaño del círculo refleja la influencia de ese estudio en el modelo (inversamente proporcional a la desviación estándar [DE] de ese estudio). La predicción de regresión está representada por la línea continua $(-0,01 \mathrm{~kg}[-0,02,-0,00] ; p=0,02)$.

\section{Eventos adversos}

En los 2 estudios analizados $(23,24)$, dado el tamaño de muestra similar, se reportaron eventos adversos generales, con predominio en el grupo de liraglutida comparado con el grupo placebo; y se reportó un RR de 1,26 (IC $95 \%$ : 0,94, 5,18) así como heterogeneidad $\left(\chi^{2}=4,28 ; p=0,04 ; \mathrm{I}^{2}=77 \%\right)$.

Los eventos adversos con mayor prevalencia y homogeneidad entre los estudios fueron eventos adversos gastrointestinales, principalmente náuseas, diarrea, vómito y dispepsia (Figura 4). El evento adverso de constipación se reportó con un RR de 2,20 (IC 95 \%: 1,09, 1,96) y se observó heterogenei$\operatorname{dad}\left(\chi^{2}=2,37 ; p=0,12 ; I^{2}=58 \%\right)$.

\section{Discusión}

Al agrupar los 3 ECA, con un total de 4.281 participantes, se evidencia una disminución de peso en un promedio de $5 \mathrm{~kg}$ en 20 semanas, con pérdida de peso de al menos $5 \%$ y $10 \%$ en los diferentes grupos de liraglutida en comparación con placebo. Así mismo se reporta en la revisión crítica de la evidencia realizada en 2017 , en la que la disminución de peso promedio fue de 4 a $6 \mathrm{~kg}$ (27).

En una revisión sistemática realizada por Khera y colaboradores, en la cual el desenlace por evaluar era la disminución de peso con distintos tratamientos con fármacos, uno de los 
Tabla 1. Características de los estudios incluidos

\begin{tabular}{|c|c|c|c|c|c|c|}
\hline Autor & Año & Metodología & Participantes & Intervención & Comparación & $\begin{array}{c}\text { Desenlace } \\
\text { evaluado }\end{array}$ \\
\hline Astrup & $\begin{array}{l}2012 \\
(23)\end{array}$ & $\begin{array}{l}\text { Estudio aleatorizado, doble } \\
\text { ciego, controlado con } \\
\text { placebo de } 20 \text { semanas } \\
\text { con extensión de } 2 \\
\text { años (patrocinador sin } \\
\text { cegamiento a las } 20 \text { semanas, } \\
\text { participantes/investigadores } \\
\text { al año) en } 19 \text { centros } \\
\text { de investigación clínica } \\
\text { europeos. } \\
\text { El ensayo se realizó entre } \\
\text { enero de } 2007 \text { y abril de } 2009 \text {. }\end{array}$ & $\begin{array}{l}\text { Población total: } \\
135 \text { hombres y } 429 \\
\text { mujeres }(n=564) \\
\text { Liraglutida: } 1,2 ; 1,8 ; \\
2,4 \text { o } 3,0 \mathrm{mg}(\mathrm{n}= \\
95,90,93 \text { y } 93) \\
\text { Placebo }(\mathrm{n}=98)\end{array}$ & $\begin{array}{l}\text { Liraglutida } \\
1,2 ; 1,8 ; 2,40 \\
3,0 \mathrm{mg}\end{array}$ & $\begin{array}{l}\text { Placebo, orlistat, } \\
\text { dieta y ejercicio/ } \\
\text { déficit de } 500 \\
\text { kcal por día y } \\
\text { asesoramiento } \\
\text { durante el } \\
\text { ejercicio durante } \\
2 \text { semanas antes } \\
\text { de ser asignados } \\
\text { aleatoriamente }\end{array}$ & $\begin{array}{l}\text { Disminución } \\
\text { de peso }\end{array}$ \\
\hline Pi-Sunyer & $\begin{array}{l}2015 \\
(25)\end{array}$ & $\begin{array}{l}\text { Estudio doble ciego de } 56 \\
\text { semanas con } 3731 \text { pacientes } \\
\text { que no tenían diabetes } \\
\text { tipo } 2 \text { y que tenían un IMC } \\
\text { de al menos } 30 \text { o un IMC } \\
\text { de al menos } 27 \text { si tenían } \\
\text { dislipidemia o hipertensión } \\
\text { tratada o no tratada. Se } \\
\text { asignó al azar a los pacientes } \\
\text { en una proporción de } 2: 1 . \\
\text { Estudio realizado desde el } \\
1 \text { de junio de } 2011 \text { hasta } \\
\text { el } 18 \text { de marzo de } 2013 \text { en } \\
191 \text { sitios en } 27 \text { países de } \\
\text { Europa, América del Norte, } \\
\text { América del Sur, Asia, África y } \\
\text { Australia. }\end{array}$ & $\begin{array}{l}\text { Población total: } \\
3731 \text { pacientes } \\
\text { Liraglutida } 3,0 \mathrm{mg} \\
\text { (2487 pacientes) } \\
\text { Placebo (1244 } \\
\text { pacientes) }\end{array}$ & $\begin{array}{l}\text { Liraglutida } \\
3,0 \mathrm{mg}\end{array}$ & $\begin{array}{l}\text { Placebo y dieta y } \\
\text { ejercicio }\end{array}$ & $\begin{array}{l}\text { Disminución } \\
\text { de peso }\end{array}$ \\
\hline Blackman & $\begin{array}{l}2016 \\
(24)\end{array}$ & $\begin{array}{l}\text { Estudio aleatorizado y doble } \\
\text { ciego, los participantes no } \\
\text { diabéticos con obesidad que } \\
\text { tenían AOS moderada (IAH: } \\
\text { 15-29,9 eventos } \mathrm{h} \text {-1) o grave } \\
\text { (IAH } \geq 30 \text { eventos } \mathrm{h}-1) \text {. } \\
\text { Los participantes elegibles } \\
\text { fueron asignados } \\
\text { aleatoriamente } 1: 1 \\
\text { de manera ciega y } \\
\text { centralizada (utilizando } \\
\text { un sistema telefónico o } \\
\text { web proporcionado por el } \\
\text { patrocinador) a liraglutida } \\
\text { subcutánea o placebo una } \\
\text { vez al día. } \\
\text { Ensayo realizado durante } 32 \\
\text { semanas. }\end{array}$ & $\begin{array}{l}\text { Población total: } \\
359 \text { participantes } \\
\text { Liraglutida: } 180, \\
\text { mujeres: } 51 \text { ( } 28,3 \\
\%) \text {, hombres: } 129 \\
(71,7 \%) \\
\text { Placebo: } 179, \\
\text { mujeres: } 50(27,9) \text {, } \\
\text { hombres: } 129(72,1 \\
\%)\end{array}$ & $\begin{array}{l}\text { Liraglutida } \\
3,0 \mathrm{mg}\end{array}$ & $\begin{array}{l}\text { Placebo y } \\
\text { complemento de } \\
\text { la dieta (déficit } \\
\text { de } 500 \text { kcal día } \\
\text {-1) y ejercicio }\end{array}$ & $\begin{array}{l}\text { Disminución } \\
\text { de peso, } \\
\text { reducción de } \\
\text { la gravedad } \\
\text { de AOS }\end{array}$ \\
\hline
\end{tabular}


Figura 2. Forest plot de comparación: disminución de peso con liraglutida frente a placebo.

\begin{tabular}{|c|c|c|c|c|c|c|c|c|c|c|c|}
\hline \multirow[b]{2}{*}{ Estudio } & \multicolumn{3}{|c|}{ Liraglutida } & \multicolumn{3}{|c|}{ Placebo } & \multicolumn{3}{|c|}{ Diferencia de medias } & & \\
\hline & Media & $\mathrm{DE}$ & Total & Media & DE & Total & Peso & $\mathrm{DE}$ & IC $95 \%$ & & \\
\hline Astrup, 2012 (23) & $-7,8$ & 7,5 & 93 & -2 & 7,5 & 98 & $9,30 \%$ & $-5,8$ & $-7,93 ;-3,67$ & $\longrightarrow$ & \\
\hline Blackman, 2016 (24) & $-6,7$ & 0,5 & 180 & $-1,9$ & 0,4 & 179 & $49,30 \%$ & $-4,8$ & $-4,89 ;-4,71$ & 】 & \\
\hline Pi-Sunyer 2015 (25) & $-8,4$ & 7,3 & 2487 & $-2,8$ & 6,5 & 1244 & $41,30 \%$ & $-5,6$ & $-6,06 ;-5,14$ & + & \\
\hline Total (IC $95 \%)$ & & 2760 & & & 1521 & & $100 \%$ & $-5,22$ & $-5,95 ;-4,50$ & & \\
\hline \multirow[t]{2}{*}{ Heterogeneidad } & $\tau^{2}=0,27$ & $x^{2}=11,89$ & $p=0,003$ & $\left.\right|^{2}=83 \%$ & & & & & & & \\
\hline & & & & & & & & & & -4 & $\begin{array}{ll}1 & 1 \\
2 & 4\end{array}$ \\
\hline
\end{tabular}

Figura 3. Metarregresión univariada de los efectos aleatorios entre liraglutida, orlistat más ejercicio y dieta, y la diferencia media de peso en kg FFM (fat free mass) entre los grupos.

\begin{tabular}{|l|c|c|c|c|c|}
\hline \multirow{2}{*}{ Modelo } & \multicolumn{5}{|c|}{ Disminución de peso } \\
\cline { 2 - 6 } & $\begin{array}{c}\text { Coeficiente (IC } \\
\mathbf{9 5 \% )}\end{array}$ & $\mathbf{T}^{2}$ & $\begin{array}{c}\mathbf{R}^{2} \\
\text { ajustado }\end{array}$ & $\mathbf{I}^{\mathbf{2}}$ & $\begin{array}{c}\text { Valor } \\
\boldsymbol{p}\end{array}$ \\
\hline Sin covariables & $2,49(0,64-4,33)$ & 6,05 & & $33 \%$ & 0,01 \\
\hline Liraglutida & $5,77(2,96-7,14)$ & 5,77 & $5 \%$ & $31 \%$ & 0,40 \\
\hline $\begin{array}{l}\text { Orlistat + dieta } \\
\text { y ejercicio }\end{array}$ & $0,31(00,9-5,77)$ & 3,16 & $40 \%$ & $41 \%$ & 0,54 \\
\hline Placebo & $0,88(0,18-1,33)$ & & & & 0,23 \\
\hline
\end{tabular}

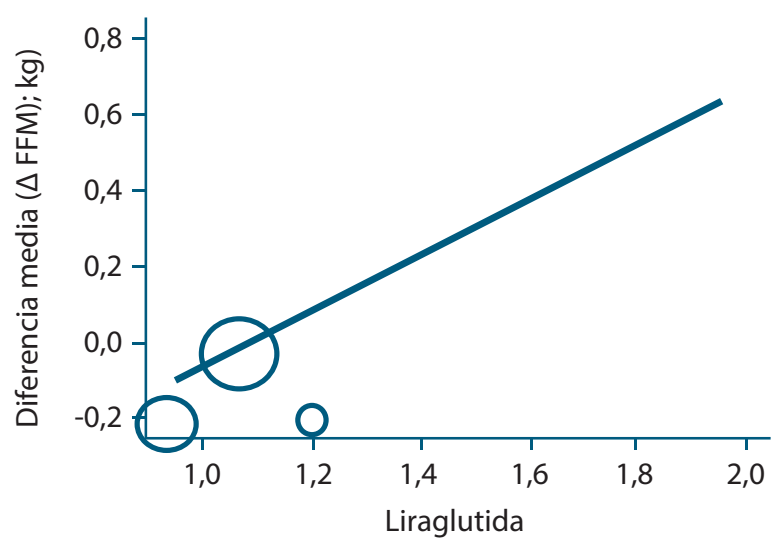

resultados obtenidos fue que la fentermina-topiramato y liraglutida se asociaron con las mayores probabilidades de lograr al menos un $5 \%$ de pérdida de peso: una pérdida de $8,8 \mathrm{~kg}$ (IC $95 \%$ : -10,20 a -7,42 kg) para fentermina-topiramato y de 5,3 kg (IC $95 \%$ : -6,06 a -4,52 kg) para liraglutida (28).
Sin embargo, al realizar tanto el metaanálisis como la metarregresión se evidenció que no hay una significancia estadística de la evidencia por la heterogeneidad de los estudios evaluados en el desenlace principal (disminución de peso), debida principalmente a las diferencias en los tamaños de muestra de cada uno de los estudios.

Por su parte, los eventos adversos representan gran importancia en el uso de la liraglutida, ya que, a pesar de ser leves con predominio de los síntomas gastrointestinales, se reportó homogeneidad en los estudios evaluados, y esto es factor importante porque significa que el uso de liraglutida genera mayor riesgo de presentar estos síntomas que el placebo.

A pesar de que los eventos adversos graves reportados en los estudios no presentan un alto porcentaje, se debe tener precaución con el uso de la liraglutida: se debe evaluar en cada paciente candidato a esta terapia coadyuvante los antecedentes tanto personales como familiares y, con esto, reducir el riesgo de que se presenten estos efectos.

En esta revisión sistemática, cada uno de los ensayos clínicos incluidos presentó un bajo riesgo de sesgo, debido a la evaluación adecuada de la secuencia aleatoria de asignación y enmascaramiento de la misma, así como la evaluación de los demás dominios referidos en la metodología. Sin embargo, la calidad de la evidencia en general se clasifica como baja de acuerdo los estándares GRADE.

\section{Limitaciones}

Se presentaron datos incompletos como la DE por cada desenlace evaluado, por lo cual se utilizó la herramienta descrita en manual Cochrane para el cálculo de los mismos (22).

\section{Conclusiones}

Los resultados evaluados de forma individual en los estudios demuestran que el uso de liraglutida disminuye el IMC 
Figura 4. Forest plot de comparación: eventos adversos, liraglutida frente a placebo. a. Evento adverso: náuseas. b. Evento adverso: diarrea. c. Evento adverso: vómito. d. Evento adverso: dispepsia. e. Evento adverso grave.

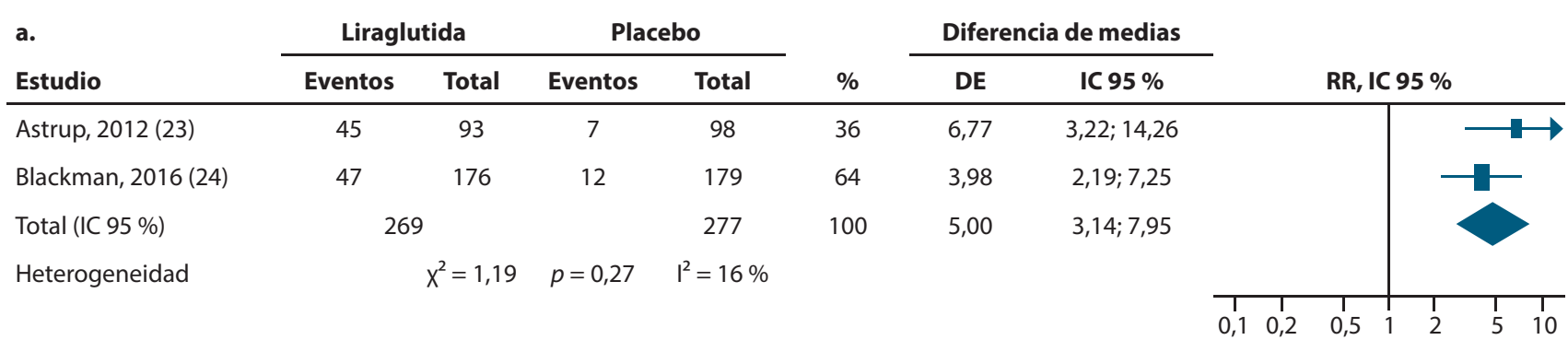

\begin{tabular}{|c|c|c|c|c|c|c|c|}
\hline \multirow{2}{*}{$\begin{array}{l}\text { b. } \\
\text { Estudio }\end{array}$} & \multicolumn{2}{|c|}{ Liraglutida } & \multicolumn{2}{|c|}{ Placebo } & \multirow[b]{2}{*}{$\%$} & \multicolumn{2}{|c|}{ Diferencia de media } \\
\hline & Eventos & Total & Eventos & Total & & DE & IC $95 \%$ \\
\hline Astrup, 2012 (23) & 14 & 93 & 10 & 98 & 41,6 & 1,56 & 0,$66 ; 3,71$ \\
\hline Blackman, 2016 (24) & 29 & 176 & 14 & 179 & 58,4 & 2,33 & 1,$18 ; 4,57$ \\
\hline Total (IC 95 \%) & \multicolumn{2}{|c|}{269} & \multicolumn{2}{|c|}{277} & 100 & 2,01 & 1,$18 ; 3,41$ \\
\hline Heterogeneidad & & $x^{2}=0,51$ & $p=0,48$ & $\mathrm{I}^{2}=0 \%$ & & & \\
\hline
\end{tabular}

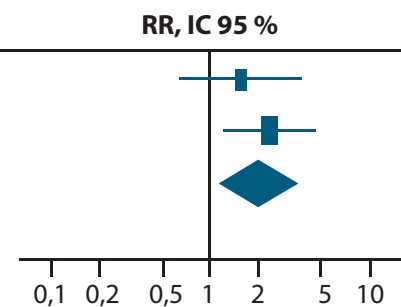

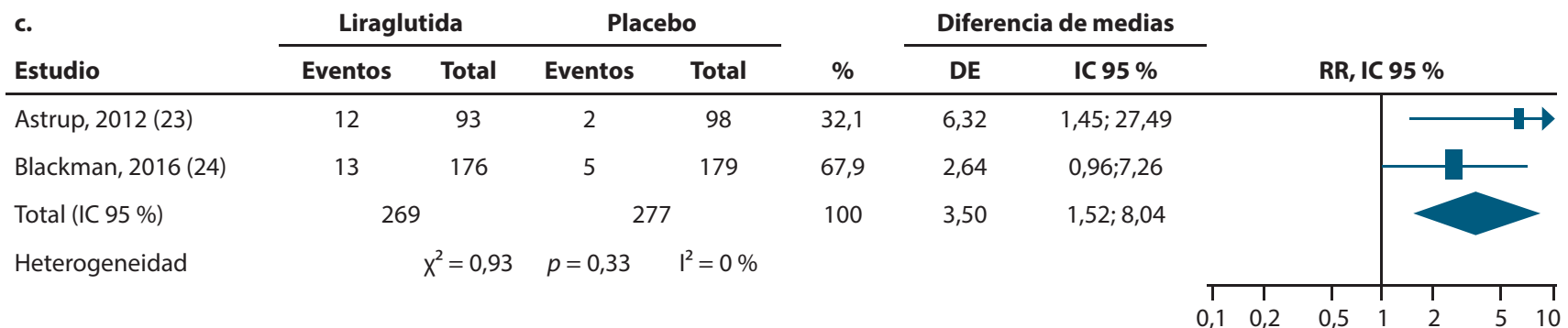

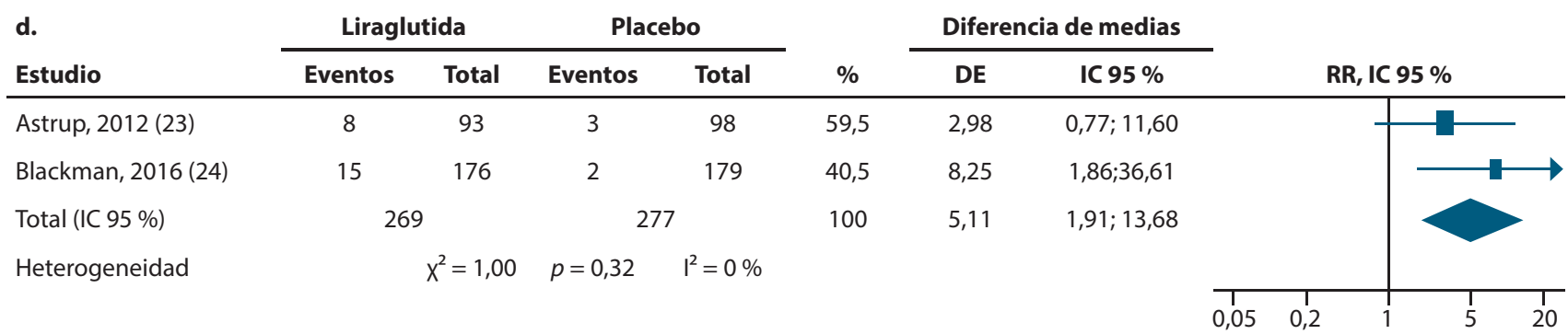

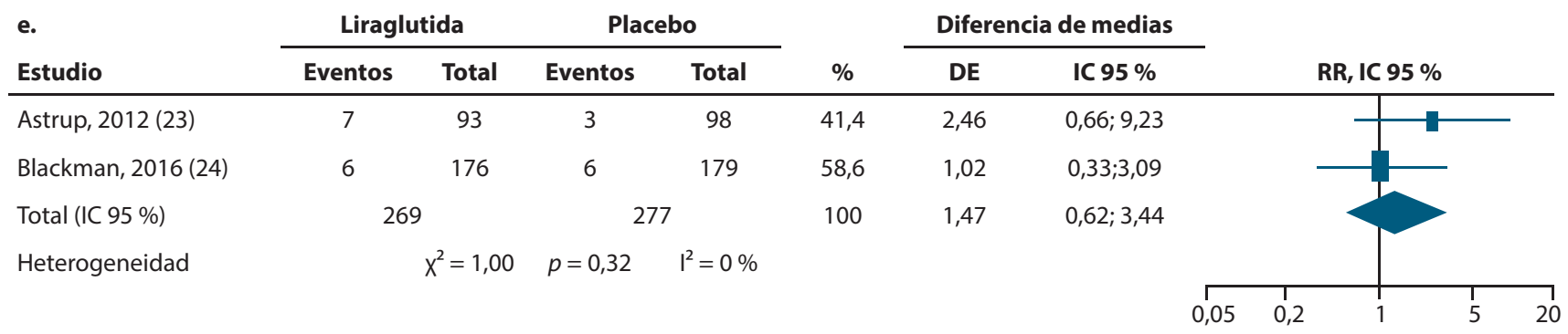

DE: desviación estándar. 
como terapia coadyuvante en personas en condición de sobrepeso y obesidad. Adicionalmente, mejora las comorbilidades asociadas con un alto IMC y disminuye el riesgo de presentar patologías que puedan causar mortalidad. Es importante con- tinuar realizando estudios primarios que evalúen esta intervención con el fin de tener mayor claridad sobre la efectividad y seguridad de la liraglutida.

\section{Referencias}

1. Obesidad y sobrepeso [internet]. OMS; 2020 [citado el 26 de mayo de 2020]. Disponible en: http://www.who.int/es/news-room/fact-sheets/ detail/obesity-and-overweight

2. Panorama de la seguridad alimentaria y nutricional en América Latina y el Caribe. Santiago de Chile: FAO, OPS, WFP, UNICEF; 2019.

3. Panorama de la seguridad alimentaria y nutricional en América Latina y el Caribe. Santiago de Chile: FAO, OPS, WFP, UNICEF; 2018.

4. Cardiovascular diseases (CVDs) [Internet]. World Health Organization; 2014 [actualizado el 1 de mayo de 2017; consultado 26 de mayo de 2020. Disponible en: https://www.who.int/en/news-room/fact-sheets/detail/ cardiovascular-diseases-(cvds)

5. le Roux CW, Astrup A, Fujioka K, Greenway F, Lau DCW, Van Gaal L, et al. 3 years of liraglutide versus placebo for type 2 diabetes risk reduction and weight management in individuals with prediabetes: a randomised, double-blind trial. Lancet. 2017;389(10077):1399-1409.

6. Frøssing S, Nylander M, Chabanova E, Frystyk J, Holst JJ, Kistorp C, et al. Effect of liraglutide on ectopic fat in polycystic ovary syndrome: A randomized clinical trial. Diabetes Obes Metab. 2018;20(1):215-218.

7. Jensen MD. Obesidad. Goldman-Cecil. En: Tratado de Medicina Interna [Internet]. Barcelona: Elsevier; 2016. p. 1458-67. Disponible en: https:// www.clinicalkey.es/\#!/content/3-s2.0 B9788491130338002202?scrollT $\mathrm{o}=\% 23 \mathrm{c} 1100$

8. Stunkard AJ, Wadden TA. Psychological aspects of severe obesity. Am J Clin Nutr. 1992;55(2 Suppl):524S-532S.

9. Wadden TA, Stunkard AJ. Social and psychological consequences of obesity. Ann Intern Med. 1985;103(6 ( Pt 2)):1062-7.

10. García Milian AJ, Creus García ED. La obesidad como factor de riesgo, sus determinantes y tratamiento. Rev Cubana Med Gen Integr. 2016;32(3).

11. Warkentin LM, Das D, Majumdar SR, Johnson JA, Padwal RS. The effect of weight loss on health-related quality of life: systematic review and metaanalysis of randomized trials. Obes Rev. 2014;15(3):169-82.

12. Mertens IL, Van Gaal LF. Overweight, obesity, and blood pressure: the effects of modest weight reduction. Obes Res. 2000;8(3):270-8.

13. Montesi L, El Ghoch M, Brodosi L, Calugi S, Marchesini G, Dalle Grave R. Long-term weight loss maintenance for obesity: a multidisciplinary approach. Diabetes Metab Syndr Obes. 2016;9:37-46.

14. Fernández-Ruiz VE, Armero-Barranco D, Paniagua-Urbano JA, Sole-Agusti M, Ruiz-Sánchez A, Gómez-Marín J. Short-medium-long-term efficacy of interdisciplinary intervention against overweight and obesity: Randomized controlled clinical trial. Int J Nurs Pract. 2018;24(6):e12690.

15. Recomendaciones mundiales sobre actividad física para la salud. Ginebra: WHO; 2010.

16. Rane SD, Gore JC. Measurement of T1 of human arterial and venous blood at 7T. Magn Reson Imaging. 2013;31(3):477-9.
17. Halawi H, Khemani D, Eckert D, O'Neill J, Kadouh H, Grothe K, et al. Effects of liraglutide on weight, satiation, and gastric functions in obesity: a randomised, placebo-controlled pilot trial. Lancet Gastroenterol Hepatol. 2017;2(12):890-899.

18. Ficha de medicamento liraglutide. clinicalkey [citado 23 de octubre 2019]. Disponible en: https://www.clinicalkey.es/\#!/content/drug monograph/6-s2.0 3496?scrollTo=\%23MonitoringParameters

19. Garber AJ, Handelsman Y, Grunberger G, Einhorn D, Abrahamson MJ, Barzilay JI, et al. Consensus statement by the American association of clinical endocrinologists and American college of endocrinology on the comprehensive type 2 diabetes management algorithm - 2020 executive summary. Endocr Pract. 2020;26(1):107-139.

20. U.S. Food \& Drug Administration (FDA). Postmarket Drug and Biologic Safety Evaluations Completed from January 2017 - March 2017. Maryland: FDA; 2017 [citado 23 de octubre 2019]. Disponible en: https://www.fda. gov/drugs/surveillance/postmarket-drug-and-biologic-safety-evaluations-completed- january-2017-march-2017.

21. Ministerio de Salud y la Protección social, Instituto Nacional de Vigilancia de Medicamentos y Alimentos (Invima). Acta No. 25 de 2015, Sesión extraordinaria virtual, 23, 24 y 25 de noviembre de 2015. Bogotá D.C: Invima; 2015.

22. Higgins JPT, Green S. Cochrane Handbook for Systematic Reviews of Interventions Version 5.1.0. The Cochrane Collaboration; 2011 [actualizado en marzo de 2011; citado 4 de mayo 2020. Disponible en: https://handbook-5-1.cochrane.org/

23. Astrup A, Carraro R, Finer N, Harper A, Kunesova M, Lean ME, et al. Safety, tolerability and sustained weight loss over 2 years with the once-daily human GLP-1 analog, liraglutide. Int J Obes (Lond). 2012;36(6):843-54.

24. Blackman A, Foster GD, Zammit G, Rosenberg R, Aronne L, Wadden T, et al. Effect of liraglutide $3.0 \mathrm{mg}$ in individuals with obesity and moderate or severe obstructive sleep apnea: the SCALE Sleep Apnea randomized clinical trial. Int J Obes (Lond). 2016;40(8):1310-9.

25. Pi-Sunyer X, Astrup A, Fujioka K, Greenway F, Halpern A, Krempf M, et al. A Randomized, Controlled Trial of 3.0 mg of Liraglutide in Weight Management. N Engl J Med. 2015;373(1):11-22.

26. Guyatt GH, Oxman AD, Sultan S, Glasziou P, Akl EA, Alonso-Coello P, et al GRADE guidelines: 9. Rating up the quality of evidence. J Clin Epidemiol 2011;64(12):1311-6.

27. Mehta A, Marso SP, Neeland IJ. Liraglutide for weight management: a critical review of the evidence. Obes Sci Pract. 2017;3(1):3-14.

28. Khera R, Murad MH, Chandar AK, Dulai PS, Wang Z, Prokop LJ, et al. Association of Pharmacological Treatments for Obesity With Weight Loss and Adverse Events: A Systematic Review and Meta-analysis. JAMA. 2016;315(22):2424-34 

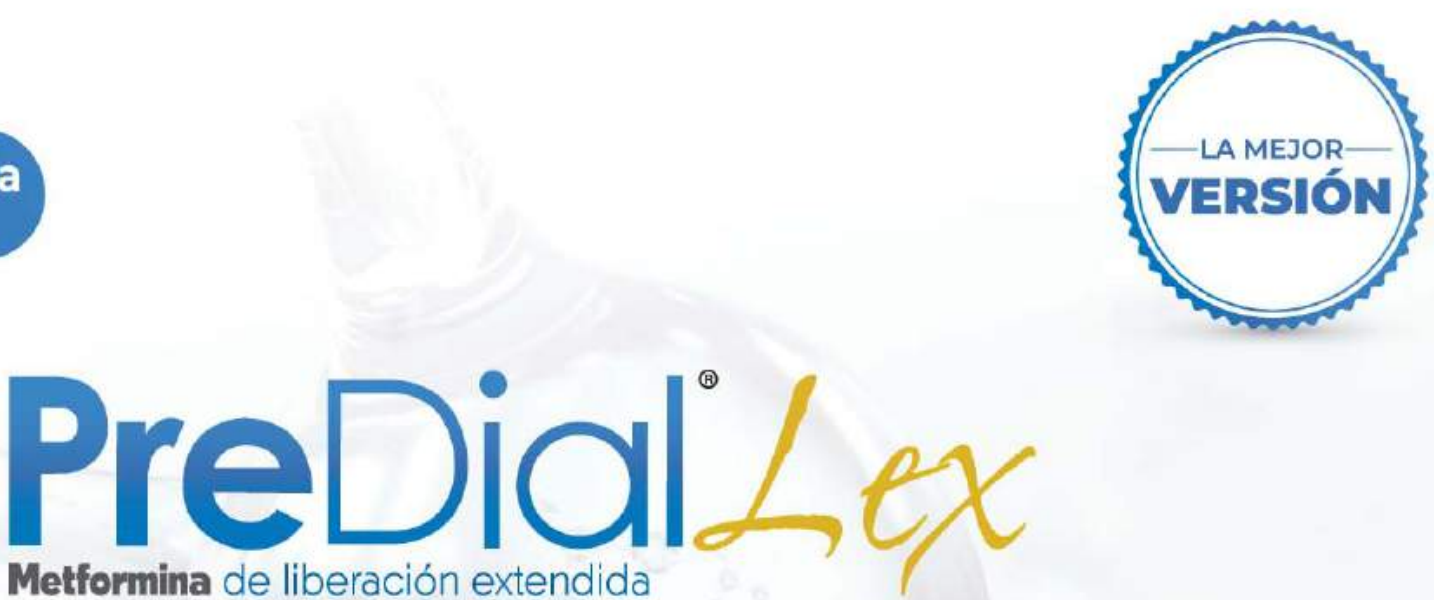

Metformina de liberación extendida

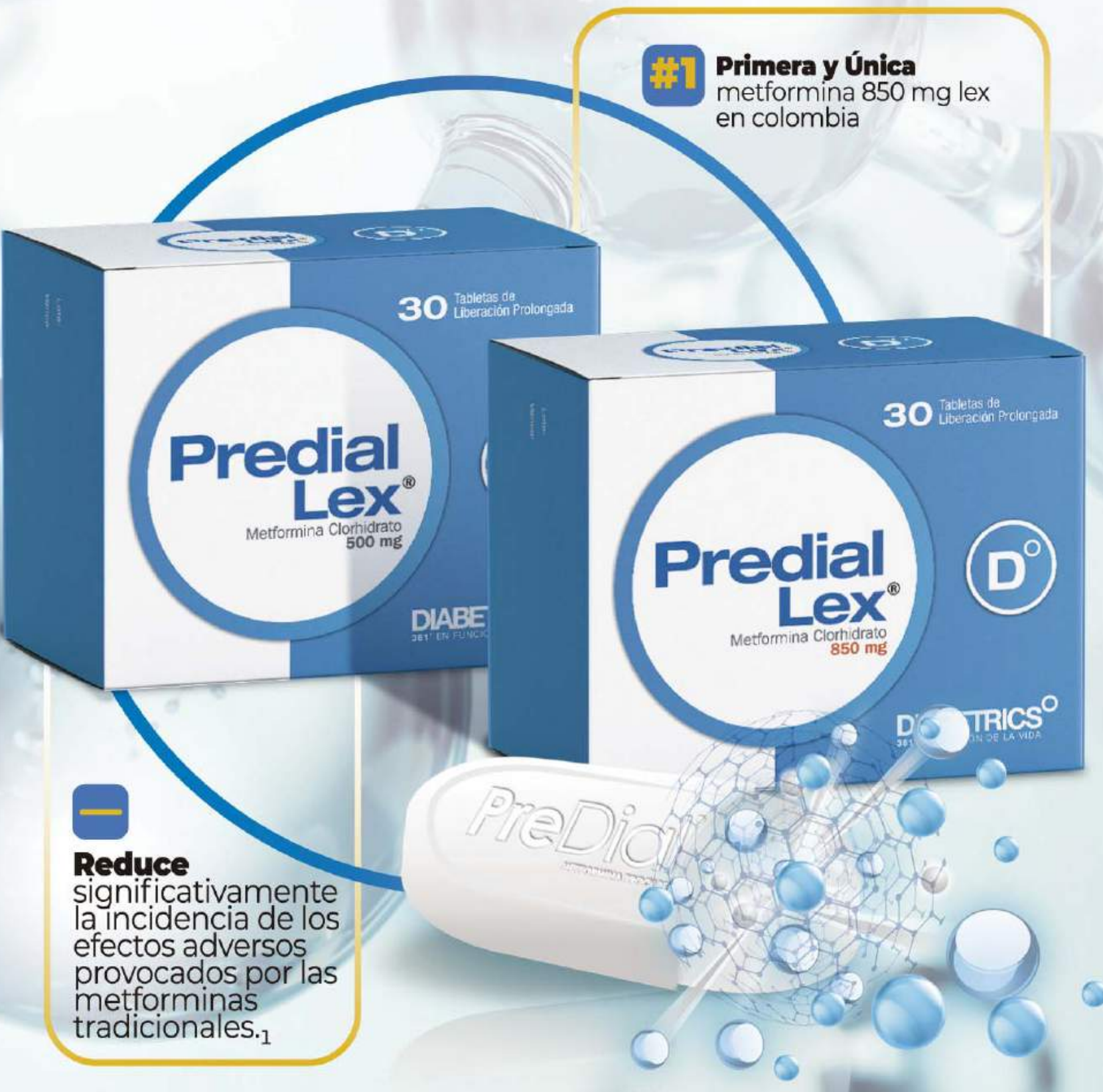

1. Blonde $L$ et al: Gastrointestinal Tolerability of extendend-release metformin tablets compared to inmediate-release metformin tablets Results of a retrospective Cohort study. Curr Med Res Opin. 2004; 20(4):565-572.W; https://doi.org/10.1185/030079904125003278 *MEDICAMENTOS DE MARCA EN CONCETRACIONES DE 850 MG, DATA MARZO 2020 CLOSE UP - LABORATORIO SILANES S.A. DE C.V. MEXICO-RS. INVIMA 2018M-0012569-RT/PREDIAL LEX 850 MG - LABORATORIO SILANES S.A. DE C.V. MEXICO-INVIMA 2012M-0013120. "Material dirigido exclusivamente para el cuerpo médico".

Indicaciones: Como adyuvante en el manejo de diabetes mellitus tipo II, que no ha respondido a medidas generales de dieta, ejercicio y sulfonamida. Alternativo en el manejo de la diabetes tipo I (insulinodependiente), según criterio del especialista. 